\section{IN BRIEF}

\section{$\Rightarrow$ VIRAL PATHOGENESIS}

\section{Rewiring the host}

Zika virus (ZIKV) is transmitted primarily to humans through Aedes mosquitoes. In the vector, infection does not lead to adverse health effects, whereas the virus can cause various symptoms and induce apoptotic cell death in humans. Thaker et al. investigated the underlying mechanism of the differential effects and showed that infection of human foreskin fibroblasts increased glucose utilization in the tricarboxylic acid cycle. By contrast, in mosquito cells, the virus increased glucose use in the pentose phosphate pathway. Infection resulted in increased AMP:ATP ratios in human cells, an effect that was not seen in mosquito cells. The change in nucleotide levels in infected human cells led to the activation of AMP-activated protein kinase-mediated signalling, which promotes apoptosis and cell death. The findings suggest that ZIKA differentially reprogrammes glucose metabolism in humans and mosquitoes, which affects host cell death and survival.

ORIGINAL ARTICLE Thaker, S. K. et al. Differential metabolic reprogramming by ZIKA virus promotes cell death in human versus mosquito cells. Cell Metab. https://doi.org/ 10.1016/j.cmet.2019.01.024 (2019)

\section{$\Rightarrow$ PARASITE BIOLOGY}

\section{Casting the net wide on malaria}

Insecticide-based strategies such as long-lasting bed nets have been very effective in controlling the spread of malaria by targeting Anopheles mosquitoes that transmit Plasmodium parasites. However, the emergence of mosquitoes that are resistant to insecticides is contributing to the resurgence of malaria. Paton et al. now provide proof of concept for a novel control strategy that involves the incorporation of the antimalarial compound atovaquone into bed nets. They coated a glass surface with atovaquone, a potent cytochrome $b$ inhibitor, and allowed female mosquitoes to rest on this surface; 6 minutes of exposure to atovaquone before or after infection with Plasmodium falciparum blocked parasite development in the mosquito midgut and prevented transmission. Thus, the addition of the antimalarial drug to insecticide-treated bed nets could be a promising transmission-blocking strategy. ORIGINAL ARTICLE Paton, D. G. et al. Exposing Anopheles mosquitoes to antimalarials blocks Plasmodium parasite transmission. Nature https://doi.org/10.1038/s41586-0190973-1 (2019)

\section{$\Rightarrow$ BACTERIAL PATHOGENESIS}

\section{New targets for leukocidins}

Staphylococcal leukocidins are pore-forming toxins that lyse leukocytes and red blood cells and cause host lethality. Lubkin et al. uncovered that those virulence factors also target endothelial cells to cause lethality. In a toxin challenge model, they showed that two leukocidins (leukocidin ED (LukED) and $\gamma$-haemolysin $\mathrm{AB}(\mathrm{Hlg} \mathrm{AB})$ ) caused vascular dysfunction and death in mice. Importantly, they found that the toxins target the Duffy antigen receptor for chemokines (DARC; also known as ACKR1) on human and mouse endothelial cells rather than leukocytes or erythrocytes. They went on to show that targeting of DARC by LukED and HlgAB impairs vascular integrity, which leads to tissue damage and organ failure, and subsequent death during acute bloodstream infection in mice. By contrast, mice with Darc-deficient endothelial cells were resistant to toxin-mediated lethality.

ORIGINAL ARTICLE Lubkin, A. et al. Staphylococcus aureus leukocidins target endothelial DARC to cause lethality in mice. Cell Host Microbe https://doi.org/10.1016/j.chom. 2019.01.015 (2019)

FURTHER READING Spaan, A. N., van Strijp, J. A. G. and Torres, V. J. Leukocidins: staphylococcal bi-component pore-forming toxins find their receptors. Nat. Rev. Microbiol. 15, 435-447 (2017)

\title{
Life in the slow lane
}

When conditions are unfavourable, for example, owing to nutrient limitation or environmental stress, bacteria can slow or even arrest their growth. Whereas spore formation and dormancy have been studied well, comparatively little is known about other survival strategies. Two new studies, one looking at Bacillus subtilis during several months of nutrient starvation and the other looking at a rare biosphere bacterium in peat soil, show that bacteria grow minimally but maintain some activity to ensure survival.

B. subtilis can form spores when encountering starvation, but as this is a costly process only a fraction of the population sporulates and the fate of the remaining cells is unclear. To study such cells, Gray et al. incubated a sporulation-deficient mutant for 100 days in a starvation buffer that contained no carbon source and showed that a fraction of the population remained viable.
Within two days of starvation, the cells became markedly smaller and rounder, and after 2 weeks they were less sensitive to several different antibiotics, which might suggest dormancy. However, their membrane potential was similar to cells in exponential growth, and they were able to synthesize proteins. Furthermore, the cells showed a unique transcriptome profile focused on nutrient acquisition and use. Last, a cell division inhibition assay indicated that the cells divided, although with a 100 -fold slower growth rate than cells in exponential growth. B. subtilis is found in oligotrophic environments such as soil and lakes, which suggests that oligotrophic growth might be common for this bacterium in its natural environment.

In the second study, Hausmann et al. indeed look at the growth of an environmental bacterium, specifically, 'Candidatus

\section{FUNGAL PATHOGENESIS}

\section{Malassezia restricta plays CARDs in the gut}

The intestinal mycobiome has been suggested to have a role in the pathogenesis of inflammatory bowel diseases such as Crohn's disease, but data are scarce. An S12N polymorphism in the gene encoding CARD9 (CARD $9^{512 \mathrm{~N}}$ ), a signalling adaptor essential for anti-fungal innate immunity, is a risk factor for Crohn's disease. Limon et al. now suggest that, in patients with Crohn's disease and CARD9 ${ }^{512 N}$, the commensal skin yeast Malassezia restricta associates with the intestinal wall and also that it promotes the production of inflammatory cytokines in myeloid phagocytes in a CARD9-dependent manner.

The authors sequenced fungal ribosomal DNA in faecal-free mucosal washings collected from the sigmoid colon and caecum of healthy individuals and patients with Crohn's disease. Compared with healthy individuals, samples from patients with Crohn's disease were enriched in the genera Malassezia, Cladosporium and Aureobasidium but depleted of the genus Fusarium.

As Malassezia were present in the gut as $65 \% \mathrm{M}$. restricta and $34 \%$

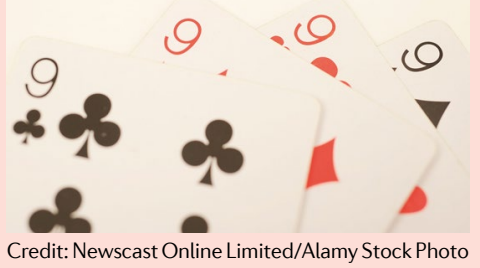

